KATHA, Vol. 11, 39-55 (2015)

\title{
CAUSAL POWER AS A STRUCTURAL IDEA: A COMPARATIVE STUDY
}

\author{
Szu-Ting Chen \\ National Tsing Hua University, Taiwan
}

\begin{abstract}
How do we typically construct a causal explanation of a target phenomenon? If we regard the phenomenon as a result of an interaction between two objects (or events), normally we would explain it by positing one of the objects (or events) as a factor that has the power to cause a change in the other object (or the other event) so that the change is regarded as the effect. This image of causal explanation is generally regarded as being Western in that it follows from Aristotle's classical idea of causation which maintains that causation results in a change between material and form of entity in the world. In China, however, the classical idea of causation involves other elements of which individual entity is only one of the components. In addition to an entity's power of causation and a resulting change in the material and form of the entity, Chinese thinkers suppose that, to make any explanation of a causal phenomenon, we must consider a number of essential components: other relevant entities in the world, their powers and their relations with the entity in question. These two traditions present different focal points in the idea of causation. But is there a chance that these two positions can be moderated so that we can develop a new position that includes the best parts of these two positions? In this paper, I first introduce Aristotle's idea of causation and then describe the structural idea of causation proposed by Chinese thinkers. Then I describe a down-to-earth case of causal inquiry to show that contemporary Western philosophers of science, if we look from the perspectives of these two
\end{abstract}


traditions, can provide a moderated account by combining the best parts of these traditions.

Keywords: Causal power, capacity, structure, Aristotle's idea of causation, Chinese idea of causation

\section{Introduction}

The two traditions, Western and Chinese, present different focal points in the idea of causation. When an explanation of a causal phenomenon is called for, Westerners usually emphasize the inherent power or capacity of the entity in question and they examine how the power or capacity behaves in the process of becoming and change. For Chinese thinkers, in addition to the history of the behaviour of an individual entity, the highest priority is the structural concern of the deployment of the position of other relevant entities and the way they influence the final result of the behavioural pattern of the entity in question. The two ways of seeing the same causal phenomenon produce two explanations of a common phenomenon. Because of the two contrasting implications of these ideas, people in these two cultural traditions have adopted different attitudes toward the world.

It may seem that these different ideas of causation can be placed at two polar extreme positions, that is, Westerns focus on a singular causal power of an individual entity, and the Chinese pay much more attention to the structure of the deployment of all involved entities. Yet, is there a chance that these two positions can be moderated so that we can develop a position that includes the salient parts of these two positions?

Indeed, a moderated account is proposed by Western philosophers of science when they point out that a causal explanation is plausible only if the following two items can be identified: first, as suggested by ancient Western wisdom, we must identify the causal role played by an entity in a particular scenario in order to see how it will act in the specific context of the scenario; second, as urged by the ancient Chinese sages, the exact way that the entity in question behaves depends on which other relevant entities exist in the specific context of the scenario, which is in turn constituted by the networks that are deployed by these relevant entities. 
In what follows, I first introduce Aristotle's inherent idea of causation and then describe the structural idea of causation proposed by Chinese thinkers. Then I describe a down-to-earth case of causal inquiry to show that contemporary Western philosophers of science, if we look from the perspectives of these two traditions, can provide a moderated account by combining the most characteristic parts of these two traditions.

\section{Aristotle's idea of causation}

When they discuss various phenomena of becoming and change, Western philosophers normally focus on the external world, which contains a great many objects, and these thinkers presume that the shape, material, and power possessed by those objects can influence the objects themselves and other relevant objects. Based on these characteristics of objects, a philosopher's main task is to provide an explanation of the nature of the phenomena of becoming and change among the objects.

Aristotle, one of the main ancient Greek philosophers, has his own idea about the phenomena of becoming and change, and his idea has had a huge impact in shaping the development of Western philosophy: All the objects in the world contain simultaneously the state of "potentiality" and that of "actualization," and there is a trade-off relation between these two states. The relation originates in the fact that an object-let us call it object A-which possesses certain materials, also possesses certain exigencies for taking the form of another, relevant object (object B). In comparison with object B, to which object A intends to change, the potential status of object $A$ is still, relative to the actual status of object $\mathrm{B}$, at the stage of privation; therefore, object A craves to become object $\mathrm{B}$ and so is in the process of heading for the actualization of changing into the form of object B. It is this trade-off process between the states of potentiality and actualization that generates the phenomenon of change of the object. In other words, what the phenomenon of change manifests is in fact the developmental process that an object, with the help of the impetus of a final cause, goes from the state of potentiality (of the object in question, A) to that of actualization (of the relevant object, $\mathrm{B}$, to which object A aims to change).

With respect to the above idea, we may refer to the relevant text of Aristotle's Metaphysics: "Therefore there must be some other thing which is the proper cause of this, and by this I mean appetite or choice. For whatever a thing chiefly desires this it will do, when, insofar as it is 
potential, it is present and comes close to the thing which is capable of being acted upon. Hence, every potency endowed with reason, when it desires something of which it has the potency and insofar as it has it, must do this thing. And it has this potency when the thing capable of being acted upon is present and is disposed in a definite way; but if it is not, it will not be able to act" (Metaphysics, Book IX, Chapter 5) ${ }^{1}$.

Take, for example, the growth of an oak tree from an acorn. An acorn is an object in the world, and the form of a huge oak tree is the target that it craves (final cause); therefore, the acorn has the potential to become an oak. That is, the form of an acorn has the potential to change into the form of an oak (formal cause). In the process of change, in addition to the materials possessed by itself, the acorn absorbs other materials such as water, nutrients, and so on from its nearby environment (material cause), and the result is that it finally turns into a huge oak tree. Thus, the causal explanation of the growth of this acorn into an oak tree uses the acorn's potential or causal power. In this explanation, the potential of the acorn is the causal power. It includes the material, form, and inherent purpose of the acorn, that is, it includes three causes. Aristotle and his Western followers tend to regard the causal relation as a feature of objects in the world; therefore, to conduct an inquiry about a causal relation is tantamount to conducting an inquiry about the nature of objects in the world.

\section{The Chinese idea of causation}

How do Chinese philosophers think about causation? We can answer this question by first examining what the Chinese mean by the word shi (勢). According to François Jullien, a prominent French sinologist, the concept embedded in the word is double-sided: on the one hand, shi means the disposition of thing and also refers to the condition, position, circumstance, configuration, arrangement, or structure of things. On the other hand, it also means force, power, potential, and the movement of things. In other words, shi carries both static and dynamic connotations. It is precisely the ambivalence of the word that makes its use very different from the Western tradition because the ancient Greeks preferred the consistency of an unambiguous concept that can be used for a neutral, descriptive purpose. Moreover, the ambivalence of the shi concept differs from the constructed antitheses on which Western representation of things rests. 
The metaphysics underlying the term shi is to perceive reality as a process of ongoing transformation; this kind of metaphysics aims to urge "the kind of potential that originates not in human initiative but instead results from the very disposition of things." From this metaphysical perspective, it may seem that reality can be perceived as a particular deployment or arrangement of things to be relied on and worked to one's advantage. Therefore, all the achievements of human beings - the wonder of art, the acquisition of wisdom, victory in war, the beauty of a piece of calligraphy - lie in "strategically exploiting the propensity emanating from that particular configuration of reality, to the maximum effect possible"; the consequence is the notion of efficacy (Jullien, 1995, p. 15-16).

As mentioned earlier, shi expresses the state that falls between the static and the dynamic. In a similar vein, the intuition of efficacy also includes two ideas: the deployment or setup of things, and the propensity or tendency of things. Following this double-sided idea of efficacy, we can say that things can be arranged in a specific way so as to be disposed to be effective. In other words, every arrangement (or structure, configuration, or environment) possesses an inherent potential or propensity that is fulfilled by the efficacy of a disposition. It is based on this notion of efficacy that reality is conceived as an ongoing process of transformation.

As is also pointed out by Jullien, ancient Chinese thinkers such as Laozi was fond of applying images of water to explicate the powerful double- sided image of efficacy. Laozi mentioned: "Nothing in the world is more weak and soft than water, yet nothing surpasses it in conquering the hard and strong - there is nothing that can compare" (Laozi, section 78). ${ }^{2}$ Why is the nature of water paradoxical in that it is so strong at conquering the hard and strong even though it is the weakest and softest thing in the world? The paradox can be debugged if we examine the characteristic nature of water from the perspective of double-sided image of efficacy.

Water's power comes from two components: first, water is very flexible - therefore, very weak - in that it can yield to be arranged in any whatever way so that it can fit with whichever situation that water is in need to conquer any target object with the least difficulty. Second, through the process of this arrangement, water gradually accumulates potential power that can be triggered by the relevant situation into manifest power whenever the situation calls for. By going through the entire process, water, starting from acting as a very weak object yielding 
in whatever way to the situation, accumulates its power with the least counter-force by going along with its neighbouring situation so as to become an object displaying its powerful manifest efficacy.

This idea of reality is very different from Western thought, in that it makes no presumption, as did Aristotle and his Western followers, about any notion of a telos - a final end (or final cause) of things - and about any means-end approach to fulfil the quest for a final cause. Chinese people seek to interpret reality solely on the basis of itself, from the perspective of a single logic inherent in the actual processes in motion. This comparison generates two concepts of "opportunity." For Western thinkers, opportunity is regarded as "a fleeting chance resulting from a favourable conjunction of circumstances that prompts action and favours its success"; for Chinese sages, it is regarded as "the most suitable moment to intervene in the course of a process that has already begun (so that, such is the pressure leading up to it, ... it is not really a matter of an intervention), the moment that sees the culmination of all the potentiality gradually acquired and that makes it [the culmination] possible to derive the greatest efficacy from it" (Jullien, 2004, p. 65).

Based on the aforementioned comparison of the philosophical presumptions of the Western and Chinese traditions, we conclude this section with additional comparisons. Unlike Western philosophy, which is based on what is hypothetical and probable, Chinese philosophy focuses on what functions automatically; instead of being in favour, as Western philosophers do, a single and transcendent pole, Chinese philosophers favour interdependence and reciprocity between two poles. And unlike Western philosophy, which values the quest of more emancipated thinking (liberty) and aspires for truth, Chinese philosophy is more desirous of finding a way to go along with the (spontaneity of) nature and seeks for a peaceful coexistence with the world. With respect to whether this seemingly contextual approach is still widely held among contemporary Chinese thinkers, Jullien wrote: “... that in itself illuminates two particularly striking aspects that seem to predispose Chinese intelligence to (military) strategy: one aspect is, of course, the polarity that it perceives in all reality; the other, its keen sense of constant to-ing and fro-ing between the two poles and its awareness that the one both implies the other and turns into it... That is a compensatory reversal, but, on that very account, it is ceaselessly innovatory. It may not be at all 'dialectical,' notwithstanding the Chinese claims sometimes made today with a view to having it recognized as philosophy. Nevertheless, it reveals the reactivity of the factors in play; it has 
certainly favoured a situational approach - both contextual and predisposition - to what we would smugly call the 'efficacy' of a subject" (Jullien, 2004, pp. 191-2, emphasis added).

\section{A moderated account: a down-to-earth case study}

One immediate question arises at this juncture: is there a chance that these two positions can be moderated so that we can develop a position that includes the most characteristic or salient parts of these two positions? In what follows, I describe a down-to-earth case of causal inquiry to show that contemporary Western philosophers of science, if we look from the perspectives of these two traditions, can provide a moderated account by combining the most characteristic parts of these two traditions.

Except for the concern of intruding into people's daily lives and infringing on their privacy, governmental officials and most civilians alike generally suppose that the installation of closed-circuit TV (CCTV) will enhance the safety of local communities by providing an "extra set of eyes" to watch over the community, reassuring people of their safety and providing police valuable evidence when crimes occur. Let us examine a prominent case.

Starting in 2001, CCTV was installed to an unprecedented extent in the UK to prevent crimes that might occur in a wide ranging areas, such as estates, shopping centres, parking lots, and public transport. This policy was widely popular. According to John Denham, then the UK Home Office Minister, the background belief that supports this popularity stems from the conviction that "CCTV has repeatedly proved its effectiveness in the fight against crime and the fear of crime" (Johnston, 2001). According to this conviction, in fact, a causal hypothesis positing that there is a negative causal relation between the extent of CCTV installation and community's crime rate-it seems that, whenever there is a change in the crime rate, we can use this hypothesis to explain why.

Given that a truthful relation between theory and model is shaped by the mutual constraint between the main hypothesis and the theory's background assumptions, we must ask, with respect to a targeted concrete phenomenon in the world such as the change of crime rate in 
our CCTV example, how can we judge whether our explanation of the phenomenon is successful? According to Nancy Cartwright, a prominent philosopher of science in the Western tradition, one way to judge the validity of a causal explanation is to check whether "the hypothesis under test is to be deduced from the data plus the background assumptions" (1989, p. 147).

Based on her general principle, Cartwright suggests that we can come up with the following approach for judging the validity of a causal explanation: to decide whether an explanation of the targeted phenomenon is successful, we must check whether the proposed hypothesis is derivable from the premises that contain the data and the background assumptions that are used to support the proposed hypothesis. If, for some reason, the proposed hypothesis cannot be derived from this combination, then what requires amending, according to this approach, is neither the collected evidence nor the causal power claims embedded in the proposed hypothesis. Rather, we must revise the background assumptions with which we specified the model. ${ }^{3}$

To express the idea in a formal way, using $e$ as data (or evidence), $a$ as background assumptions, and $h$ as hypothesis, we may express Cartwright's idea in the following formal way:

$$
e+a=>h
$$

where "+" denotes "and," and "=>" denotes "derive" or "deduce." Now, suppose that we face an anomalous phenomenon that our original hypothesis $h$ cannot explain; Cartwright's approach requires us to find another plausible hypothesis for this phenomenon by revising the background assumptions with which we specify the model. In practice, this means that once we find that the new evidence regarding the phenomenon - say, $e$ '-is in conflict with the original $h$-i.e., using the formula, it means that the new evidence $e$ ' plus the original background assumptions $a$ cannot derive the original $h$ :

$$
e^{\prime}+a \neq>h
$$

where " $\neq>$ " represents "cannot derive," then we will attempt to establish a new model. To do so, we revise the original background assumptions and propose new assumptions $\left(a^{\prime}\right)$ and hope that the new assumptions $a^{\prime}$ plus new evidence $e$ ' will be able to derive a new hypothesis $h$ '; again, using the formula, we will have: 


$$
e^{\prime}+a^{\prime}=>h^{\prime}
$$

Once the derivation from $\left(e^{\prime}+a^{\prime}\right)$ to $h^{\prime}$ is confirmed, then we can use new theory T' (which contains $h^{\prime}$ and $a^{\prime}$ ) to explain, from the perspective of the original theory $\mathrm{T}$ (which contains $h$ and $a$ as its components), the originally unexplainable phenomenon (or evidence) $e$ '; in other words, $\mathrm{T}^{\prime}=h^{\prime}+a^{\prime}$ can be applied to give an explanation of the originally anomalous phenomenon $e$ '.

Consider our CCTV example (see also Cartwright and Hardie, 2012, pp. 109-112 and pp. 181-185). As pointed out earlier, it is generally supposed that there is a negative causal relation between the density of the installations of CCTV cameras and the number of car crimes in parking lots. According to the idea of causal power, we can suppose that investigators may hold a belief in the following ascription of causal power: the installation of CCTV cameras has the causal power to prevent the occurrence of car crimes. In this case, CCTV can act as a cause to prevent car crimes by "catching thieves in the act." But, be it as effective as it can be, CCTV may sometimes fail to exert such prevention. For example, when the infrastructure of a police support system for some reason cannot provide in-time support at the crime site, this fact will significantly reduce CCTV's effectiveness in deterring car crimes.

Suppose that we use the claim "CCTV can act as a cause to prevent car crimes" as our initial causal hypothesis-i.e., $h$ - which is derived from a very simple model containing only CCTV and car crimes as its two factors, with an initial set of assumptions-i.e., $a$ - including the statement that there is a well-established police support system. Next, further suppose that we observe a dramatic reduction in the car crime rate after our community installs CCTV, which is connected directly to our local police station; this observation is the evidence-i.e., $e$-for our case. By referring to our formula, $e$ and $a$ together can be used as a conglomerate to deduce $h$; that is, the evidence of a significant drop in car crimes after installing CCTV, plus the assumptions (including the assumption "there is a good police support system") can together be used to derive our initial causal hypothesis that CCTV can act as a cause to prevent car crimes. By confirming this deduction, we can use this confirmed hypothesis to provide a causal explanation for the phenomenon of the drop in car crime rate in our community. 
Suppose we now observe that, contrary to our previous observation, there are quite a few anomalous cases, all indicating that the number of car crimes is again increasing. The most convenient way to develop another causal hypothesis to explain the anomalous phenomenon- $e$ ' in our case-is to determine whether the initial set of assumptions is still sustainable. Suppose, after investigating our local police station, we find that, due to a manpower shortage, the support system is malfunctioning at the moment. We can then come up with a new set of assumptions not including "good police support system" as one component; call this new set of assumptions $a$ '. Further, the lack of a good police support system can be regarded as a new causal factor-the factor of a malfunctioning police support system - which is now included in the original simple model (which formerly contained only CCTV and car crime as its two factors). In our new model, there are three factorsCCTV, a malfunctioning police support system, and car crime-which are combined as a causal network such that CCTV has the causal power to decrease, and a malfunctioning police support system has a contrary causal power to increase, the rate of car crime; the evidence of the anomalous phenomenon of increasing car crimes- $e$ '-is the direct result derived from the fact that the contrary causal power of increasing car crime defeats the causal power of decreasing it. In this new scenario, $e^{\prime}$, together with $a$ ', can surely derive a new causal hypothesis $h$ ' that "CCTV can act as a cause to prevent car crimes only with the help of a well-established police support system."

Other assumptions (such as "the road leading from the police station to the car crime site is in good condition") can also be included as additional factors (and a further new set of assumptions, $a$ ", is formed). Such a factor will act as a cause with a contrary causal power (the cause that "the road leading from the police station to the crime site is in bad condition" will encourage car thieves to ignore the effect of the installation of CCTV) to increase car crime. With these other factors added to the original simple model, we can derive another new causal hypothesis ( $h$ '), which can be used to accommodate further anomalous phenomena $\left(e\right.$ '), and another round of causal inference-say, $e^{\prime \prime}+a$ "' $\Rightarrow h$ ' - can be conducted. In other words, by adding the contrary causal power as a supplementary premise to the original simple model, we can derive the new hypothesis from the premise that contains the following three elements: the new anomalous evidence, the old assumptions of the previous model, and the new condition ("the road is in bad condition") have the contrary causal power of increasing the car crime rate. The same 
procedure of inference continues whenever a new anomalous phenomenon occurs.

Thus, our knowledge of the targeted factors' causal powers, coupled with our knowledge of how to deploy the relation among those factors, can be regarded as the foundation on which the edifice of knowledge can be built. The entire process of causal inquiry looks like this: by adding to (or subtracting from) the original simple model additional factors that carry with themselves stable causal powers, we can investigate step by step how the net effect results from the interaction among those factors - including the new factors and the old ones.

The next question immediately arises is: whether the causal hypothesis about the causal power of a factor stated in our causal theory is external valid - that is, how we can move from "a causal hypothesis works somewhere" to "the hypothesis will also work here." According to Cartwright and Jeremy Hardie's 2012 book, in order to make sure whether a causal hypothesis about the causal power of a factor in question is externally valid, "[t]here are two further kinds of facts you will have to nail down... to build a road from 'it works somewhere' to 'it will work here.' These are... facts about the causal role the [factor in question] plays and facts about the support factors that must be in place if the policy is to work. Without warrant for these, you don't have warrant for your prediction" (2012, p. 6, emphases added).

Pawson and Tilley (1997, pp. 78-81) pointed out that there are a great many mechanisms that make it possible for CCTV to play a great many causal roles in a great many contexts containing various sets of support factors, constituting various causal structures for CCTV's effectiveness. This fact further illustrates that there is no operationalizable way to match a specific mechanism with a specific context. We return to the CCTV example to illustrate the points. Recall our hypothesis that CCTV has the causal power to prevent the occurrence of car crimes. We further propose a causal hypothesis that CCTV can act as a cause to prevent car crimes by "catching (thieves) in the act." Suppose that the city government holds the belief that CCTV has this causal power and accepts the causal hypothesis; the government proceeds to install CCTV in every parking lot to prevent car crimes. Further suppose that the implementation of the policy is quite successful in that the rate of car crime is significantly reduced after the installation.

Next, suppose that, after a long period of time, the government faces an anomalous phenomenon: the rate of car crime is again rising. How can we offer an explanation for such a phenomenon? According to 
Cartwright and Hardie's account, the anomalous phenomenon is triggered by the insufficiency of the factors that, combined jointly, will bake a "causal cake" that can be used to give a causal explanation of the phenomenon. As mentioned earlier, without the help of other support factors, CCTV cannot reliably prevent car crimes. Therefore, according to Cartwright and Hardie's metaphor, the factor of CCTV installation and these other support factors are then supposed to be the slices-i.e., the components - that jointly constitute the causal cake of the effectiveness of CCTV installation. It may thus seem that CCTV, a good police support system, and good road conditions are three factors that are jointly sufficient to prevent car crimes, whereas CCTV, by itself, is an insufficient but necessary part of this jointly sufficient set of factors. CCTV is, in the acronym coined by J. L. Mackie (1980), an INUS condition-i.e., an Insufficient but Necessary part of an Unnecessary but Sufficient condition - to the effectiveness of CCTV installation. When a cause is an INUS condition for an effect, specifying a set of INUS conditions is thus equivalent to identifying a causal structure (Hoover, 1990) or a causal cake (Cartwright \& Hardie, 2012, p. 62-68).

Let's consider another scenario of our CCTV story to illustrate the importance of identifying the causal role played by the factor in question in our explanation of a causal phenomenon. Suppose further that the city government discovers that no adequate police support system exists and most of the roads connecting police stations with various parking lots are in bad condition-that is, most of the slices that constitute the causal cake of the effectiveness of CCTV are missing from the list of the three independently necessary but insufficient factors (including CCTV, a good police support system, and good road conditions); will this fact lead the city government to regard installation of CCTV as an unworkable policy for preventing car crimes? Not necessarily! Even though the lack of good roads and the malfunctioning of a police support system indeed reduce in a certain way the effectiveness of the policy, the policy can still have the causal power to exert its efficacy in another way.

The idea is this: even though CCTV has the causal power to deter car crimes, each specific scenario requires that CCTV play a specific causal role. CCTV does not have an intrinsic property of exerting inhibiting power; rather, the way it exerts its power depends on the specific requirement of the concrete case where it is installed. For example, in the first scenario, CCTV can be used as a hidden camera to record thieves in the act of stealing cars and notify the police, who then 
catch the criminals on site. Our sufficient set of necessary factors, which includes CCTV as an INUS condition, and an adequate police support system and good road conditions as two support factors (or, to use our terminology, two background conditions) jointly constitute a causal structure within which CCTV exerts its preventing power. Or, in the second scenario, CCTV can be used as a visible device to frighten criminals away from the sites where cameras are installed. By acting with the causal role as a visible device, the jointly sufficient set of factors for the first case is not necessary for CCTV to be effective in reducing car crimes in the second. In the second case, CCTV needs another set of background conditions to sustain its effectiveness, for example, it needs the background condition that CCTV evidence must be admissible in court so that would-be thieves will be deterred by their worry of being recorded.

Therefore, there are two causal cakes, each of which contains CCTV as its main causal factor-its INUS condition-along with other support factors. Each combination of the slices of the relevant factors will lead to the result of the effectiveness of CCTV installation. As indicated in my explanation of the strategy and in our CCTV example, let's call "sometime" in "a policy works sometime" $t_{1}$, and call "another time" in "the policy will also work another time" $t_{2}$. Suppose further that we want to make an inference by checking whether a policy that works in $t_{1}$ will also work in $t_{2}$. To do that, we must ensure, first, that the causal role played by the policy in $t_{1}$ will be the same as it plays in $t_{2}$; and, second, we need to ensure that there are sufficient factors-in our terminology, sufficient background conditions - in place to support the policy to produce the targeted effect. Only when we have ascertained the causal role the policy plays in both $\mathrm{t}_{1}$ and $\mathrm{t}_{2}$ can we then determine which background conditions are needed with respect to the corresponding causal role, and whether they are indeed present. By undertaking this analysis, we have obtained an inference ticket that warrants a successful inference of our conclusion in $t_{1}$ and $t_{2}$.

To be more precise, to ensure that the result derived from the exertion of a causal factor's power in $t_{1}$ can be projected to another concrete scenario in $t_{2}$, the first step is to apply the procedure suggested in this paper to check whether the causal role played by the policy is still the same in the new scenario. If we are lucky, we will discover that the two causal roles are the same; in that case, we can then begin to check whether, in the new scenario, the same background conditions exist to help enable the policy to produce the targeted effect. If both checks are 
passed, then the causal claim derived from the original scenario can be used to explain the targeted effect in the new scenario at $t_{2}$. On the other hand, if, for some reason we are not so lucky, we may discover that the policy does not play the same causal role in the new scenario at $t_{2}$; we then conduct a search to discover the causal role the policy plays in the new scenario at $t_{2}$. Additionally, we should begin another search to discover a different set of background conditions to support the policy in its new causal role, to produce the same policy effect.

\section{Conclusion}

The examination of Cartwright's causal inquiry approach shows that, in contemporary Western philosophy of science, there is a convergence of the Chinese and West ideas of causation. As is indicated in section on Aristotle's idea of causation, when conducting a causal inquiry, Westerners tend to focus on the inherent power of an entity and to examine how the power behaves in the process of becoming and change. In other words, Westerners focus on the task of identifying a singular causal power of an individual entity. This characteristic of Western idea of causation is prominent in Cartwright's approach. When Cartwright emphasizes that, with respect to each specific scenario, identifying the causal role played by the factor in question is the first priority task that needs to be conducted so that the set of other supportive factors can thus be determined, what she really follows is the Aristotle's idea of causation since the ancient Greek time. On the other hand, when Cartwright points out that CCTV does not have an intrinsic property of exerting inhibiting power to deter car crimes, the way it exerts its power depends on the specific deployment of other relevant factors of a concrete case where it is installed, what she indicates is in fact a structural idea of causation that has long been urged by Chinese thinkers in their tradition - that is, the structural idea of efficacy that every arrangement of the relevant factors possesses an inherent potential that is fulfilled by the efficacy of a disposition which is in turn triggered by the arrangement itself.

Following from the characterization of the West and Chinese ideas of causation in general and the result we collect from our case study in specific, it would not be an exaggeration to say that the Western idea of causation, due to its emphasis on means-end approach for 
fulfilling the quest for a final cause, aims to call for a prompt action to actively intervene in the on-going process of the world. On the other hand, instead of favouring an active intervention, the Chinese idea of causation, due to its focus on the structural aspect of the macroscopic arrangement of relevant factors, tends to suggest a passive attitude toward the most suitable moment for intervention in the course of a process in the world. If Cartwright's causal inquiry approach is a representative case of the foremost methodology that sits in the frontier of the methodological discussion in contemporary Western philosophy of science, then its hybrid feature that combines, one the one hand, static and dynamic notions of causation; and, on the other hand, active and passive attitudes toward human intervention in the on-going process of the world, should prove that the combination of the most characteristic parts of the West and Chinese traditions should be regarded as a plausible way for conducting academic research in the forthcoming years.

\section{Endnote}

${ }^{1}$ The quotation is a version of translation excerpted from the website at: http://dhspriory.org/thomas/Metaphysics9.htm\#3, which I think is more precise and appropriate to our current context. For the more classic translation, please refer to Aristotle: Metaphysics, Book I-IX (Loeb Classical Library No. 271), translated by Hugh Tredennick. Cambridge, MA: Harvard University Press, 1933: "Therefore there must be some other deciding factor, by which I mean desire or conscious choice. For whichever of two things an animal desires decisively it will do, when it is in circumstances appropriate to the potency and meets with that which admits of being acted upon. Therefore everything which is rationally capable, when it desires something of which it has the capability, and in the circumstances in which it has the capability, must do that thing. Now it has the capability when that which admits of being acted upon is present and is in a certain state; otherwise it will not be able to act."

${ }^{2}$ The quotation is a version of translation retrieved from the website at: http://www.indiana.edu/ p374/Daodejing.pdf. For Jullien's own translation, please refer to Jullien, F., 2004, p. 171: "In the whole world there is nothing more flexible or weaker than water, but to attack that which is hard and strong, nothing can 'surpass' it-or 'replace' it either."

${ }^{3}$ Cartwright's approach for causal inquiry is widely known as a type of bootstrap method for causal inference, which is inspired by Clark 
Glymour's bootstrap theory of confirmation (Glymour, 1980). For more detailed explanation of the inferential method advocated by Cartwright, please refer to my paper: Chen, S. (2009). Manoeuvring between theory and fact: A case study of economic modelling. Taiwanese Journal for Studies of Science, Technology and Medicine 9: 57-121; the discussion of the method appears in pp. 71-76.

\section{References}

Aristotle. (n.d) Metaphysics, Book IX, Chapter 5. Retrieved from http://dhspriory.org/thomas/Metaphysics9.htm\#3

Aristotle. (1933). Metaphysics, Book I-IX (Loeb Classical Library No. 271), translated by Hugh Tredennick. Cambridge, MA: Harvard University Press.

Cartwright, N. (1989). Nature's capacities and their measurement. Oxford, UK: Oxford University Press.

Cartwright, N. \& Hardie, J. (2012). Evidence-based policy: A practical guide to doing it better. Oxford, UK: Oxford University Press.

Chen, S. (2009). Manoeuvring between theory and fact: A case study of economic modelling. Taiwanese Journal for Studies of Science, Technology and Medicine 9: 57-121. (in Chinese)

Glymour, C. (1980). Theory and Evidence, Princeton, NJ: Princeton University Press.

Hoover, K. (1990). The logic of causal inference: Econometrics and the conditional analysis of causation. Economics and Philosophy 6 (2): 207-234.

Johnston, P. (2001). £80m fund to put Britain on CCTV. Telegraph. Retrieved from http://www.telegraph.co.uk/news/uknews/ 1338047/80m-fund-to-put-Britain-on-CCTV.html

Jullien, F. (1995). The propensity of things: Toward a history of efficacy in China. New York, USA: Zone Books.

Jullien, F. (2004). A treatise on efficacy: Between Western and Chinese thinking. Honolulu, USA: University of Hawaii Press.

Laozi. (n.d) Daodejing. Retrieved from http://www.indiana.edu/ $\sim$ p374/Daodejing.pdf

Mackie, J. L. (1980). The cement of the universe: A study of causation. Oxford, UK: Oxford University Press.

Pawson, R., \& Tilley, N. (1997). Realistic evaluation. London: SAGE Publication. 


\section{Acknowledgement}

I would like to express my gratitude to those who helped me shape the present form of this paper. I express my gratitude to my colleague from the economics department, Professor Hsiang-Ke Chao, for his helpful discussion and comment on the case study presented in the paper. This paper was presented in_Seminar on Malay and Chinese Philosophy of Sciences held at the Centre for Civilisational Dialogue in June 2014, University of Malaya, Kuala Lumpur, Malaysia and received helpful comments from the audiences, I should also express my thanks to them. Finally, I have benefited much from the helpful comment offered by an anonymous referee; the suggestion and guidance helped me greatly improving the previous argumentative structure of the paper, and so my great appreciation also goes to the referee. This paper is the result of a research project supported by Ministry of Science and Technology, Taiwan, project number: NSC-102-2420-H-007-001-MY3. 
KATHA - The Official Journal of the Centre for Civilisational Dialogue 